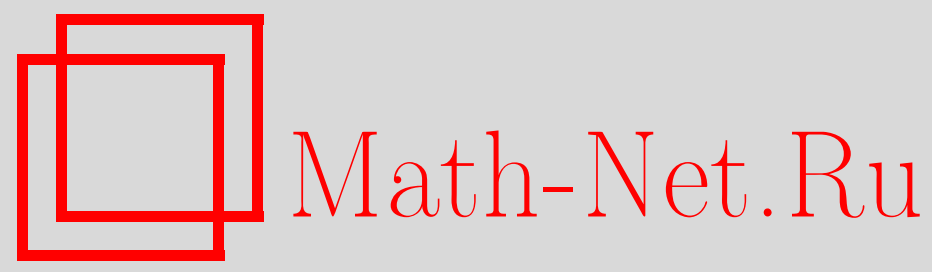

И. Д. Кан, Б. С. Стечкин, И. В. Шарков, К проблеме Фробениуса трех аргументов, Матем. заметки, 1997, том 62, выпуск 4, 626-629

DOI: https://doi.org/10.4213/mzm1646

Использование Общероссийского математического портала Math-Net.Ru подразумевает, что вы прочитали и согласны с пользовательским соглашением http://www . mathnet.ru/rus/agreement

Параметры загрузки:

IP : 54.209 .52 .79

26 апреля 2023 г., 13:19:03

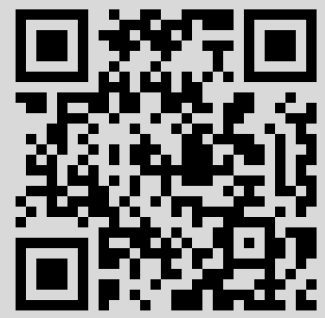




\section{К ПРОБЛЕМЕ ФРОБЕНИУСА ТРЕХ АРГУМЕНТОВ}

\section{И. Д. Кан, Б. С. Стечкин, И. В. Шарков}

Пусть $a_{1}, a_{2}, \ldots, a_{t}$ - взаимно простые натуральные числа и пусть $g=g\left(a_{1}, a_{2}, \ldots, a_{t}\right)$ - наибольшее целое $g$, не представимое в виде

$$
g=a_{1} x_{1}+a_{2} x_{2}+\cdots+a_{t} x_{t}, \quad x_{i} \in \mathbb{N}_{0}=\{0,1,2, \ldots\}
$$

Для $t=2$ имеется классический результат Сильвестра [1]: $g\left(a_{1}, a_{2}\right)=a_{1} a_{2}-a_{1}-a_{2}$.

При $t \geqslant 3$ вопрос существенно усложняется и носит название проблемы уса. Так при $t=3$ известны алгоритмы, дающие точное решение за $O\left(\ln a_{1}\right)$ арифметических операций (см., например, [2]), но мало что известно о существовании (или отсутствии) формулы, решающей ту же проблему за заранее ограниченное количество действий. Между тем, со времени работы Джонсона (1960) регулярно появляются работы, авторы которых предлагают свои алгоритмы для вычисления $g$. Появление таких работ оправдано по двум причинам. Во-первых, новые алгоритмы появляются, как правило, из новых методов, которые могут, в конечном итоге, привести к новьм результатам. Во-вторых, есть надежда, что из какого-нибудь алгоритма удастся вьвести точную формулу функции Фробениуса. Новому методу вычисления $g(a, b, c)$ посвящена данная заметка.

Интересно, что этот метод возник с “компьютерной подсказки”. Так именно пакетный диалог с компьютером подсказал в 1992 году следуюшие частные и достаточно общие формулы:

$$
g(n, n+1, n+2)=\left[\frac{n}{2}\right] n-1
$$

(см. $[3$, c. $49-50])$,

$$
\begin{gathered}
g(n, n+1, n+3)=\left[\frac{n}{3}\right](n+1)+2\left[\frac{n+1}{3}\right]-1, \\
g(n, n+1, n+4)=\left[\frac{n}{4}\right](n+1)+3\left[\frac{n+1}{4}\right]+n-1,
\end{gathered}
$$

исходя из которых общий вид правых частей просматривается в виде функции

$$
\Phi(n, p)=\left[\frac{n}{p}\right](n+1)+(p-1)\left[\frac{n+1}{p}\right]+(p-3) n-1,
$$

(см. [3, с. 99]), однако уже при $p=5$ имеем $g(n, n+1, n+5)=\Phi(n, 5)-n \delta(n, 6)$, где $\delta-$ дельта Кронекера.

Вычисление $g(a, b, c)$ можно свести к нахождению $g(n, n+1, n+p)$.

Работа выполнена при частичной поддержке Российского фонда фундаментальных исследований, грант № 96-01-00492. 
ТЕОРемА 1. Пусть $d$ - наибольший общий делитель $а$ и $b, a<b<c$, тогда

$$
g(a, b, c)=\frac{a b(g(n, n+1, n+p)+2 n+1)}{d n(n+1)}+(d-1) c-(a+b),
$$

əде $n=\frac{b}{d} v-1, p=n\left(\frac{c d v}{a}-1\right)$, a $v \in \mathbb{N}$ таково, что $b v \equiv d(\bmod a), v d<a$.

Данное утверждение получается путем трехкратного использования формулы Джонсона $g(x, y, z)=l g(x / l, y / l, z)+(l-1) z[2]$.

Требует отдельного обоснования вычислительная целесообразность формулы (1). Предположим, что мы составили таблицу значений $g(n, n+1, n+p)$ для многих (грубо говоря, для всех) $n$ и $p$. В этом случае можно показать, что вычисление $g(a, b, c)$ по формуле (1) проводится асимптотически быстрее, чем по алгоритму Джонсона. Это связано с тем, что алгоритм Джонсона требует $O(\ln a)$ умножений и делений и, следовательно, $O\left(\ln ^{3} a\right)$ единиц времени. Вычисление параметров $d$ и $v$, которьми определяется сложность формулы (1), можно осушествить за $O(\ln a)$ простейших арифметических действий (таких как сложение, вычитание, деление на 2 , умножение на 2). Полезно заметить, что в рамках [4] отношение трудоемкости умножения $k$-значных чисел к трудоемкости их сложения равно величине $C_{k} \log _{2} k\left(\lim _{k \rightarrow \infty} C_{k}=C\right.$, где $C$ - абсолютная константа). Следовательно, вычисления, проводимые по формуле (1), асимптотически в $C \log _{2} \log _{2} a$ раз быстрее алгоритма Джонсона (за счет априорного знания $g(n, n+1, n+p))$. По поводу общей алгоритмической сложности проблемы Фробениуса см. [5, с. 106-108].

Регулярную область точных решений дает

TeOpema 2. Пусть $n, p \in \mathbb{N}, \Psi(n, p)=\min \{\Phi(n, 1), \Phi(n, p)\}$, moгдa

$$
g(n, n+1, n+p) \leqslant \Psi(n, p),
$$

причем в (2) равенство имеет место тогда и только тогда, когда

$$
\frac{n+1}{p-1} \geqslant \min \left\{\left[\frac{n-1}{p}\right]+1, \frac{1}{[p / n]}\right\}
$$

СлЕДСТвИЕ 1. Если $n \geqslant p(p-4)+2, \operatorname{mo} g(n, n+1, n+p)=\Phi(n, p)$.

Ясно, что если $c \geqslant g(a, b)+1$, то $g(a, b, c)=g(a, b)$, а это влечет

СлеДСтвИЕ 2. Если $n \leqslant 1+\sqrt{p+1}$, mo $g(n, n+1, n+p)=n^{2}-n-1$.

Суть доказательства составляет не более чем одношаговое применение алгоритма Джонсона. Нерегулярность в классе точных решений проявляется при невьполнении (3). Эта область характеризуется тем, что в ней всегда

$$
g(n, n+1, n+p)=\Psi(n, p)-(\alpha n+\beta(n+1)), \quad \alpha, \beta \in \mathbb{N}_{0},
$$

однако эту двумерную (от $\alpha$ и $\beta$ ) таблицу разностей $\Psi-g$ удается выразить однопараметрически. 
ТЕОРемА 3. Для любых $n, p \in \mathbb{N}$ существует натуральное $k(1 \leqslant k \leqslant n(n-1) / 2)$ makoe, чmo

$$
g(n, n+1, n+p)=\Psi(n, p)-M_{n}(k),
$$

где $M_{n}(k)=[\sqrt{2 k}-0.5] n+k-1-0.5[\sqrt{2 k}-0.5][\sqrt{2 k}+0.5]$.

Это дает точное решение в части нерегулярной области.

СлЕдСТВиЕ 3. Если $n \geqslant p-2 u$

$$
\frac{1}{2}\left[\frac{2 n-1}{p}+1\right] \leqslant \frac{n+1}{p-1}<\left[\frac{n-1}{p}+1\right],
$$

mo

$$
g(n, n+1, n+p)=\Phi(n, p)-\min \left\{\left(p-2-\left[\frac{n}{p}\right]-p\left\{\frac{n}{p}\right\}\right) n, p\left\{\frac{n}{p}\right\}(n+1)\right\} .
$$

Стало быть, если

$$
n \geqslant\left[p\left(\frac{p-6}{2}\right)+2\right]
$$

то теорема 2 со следствием 3 дают для $g(n, n+1, n+p)$ точные формулы.

Для малых $n \leqslant p-3$ одношаговое применение алгоритма Джонсона дает

СлЕДСТВИЕ 4. Если $n \leqslant p-3 u$

$$
0<\left[\frac{n-1}{n\{p / n\}}+1\right]\left(n\left\{\frac{p}{n}\right\}-\left[\frac{n+p}{n}\right]\right) \leqslant n+1,
$$

mo

$$
\begin{aligned}
g(n, n+1, n+p)=( & +1)\left(n\left\{\frac{p}{n}\right\}-1\right)-n+\left[\frac{n-1}{n\{p / n\}}\right](n+p) \\
& -\min \left\{(n+1)\left(\left[\frac{n-1}{n\{p / n\}}+1\right] n\left\{\frac{p}{n}\right\}-n\right), n+p\right\} .
\end{aligned}
$$

Наконец, комбинирование полученных точных решений с алгоритмом Джонсона и $M_{n}(k)$, дает новый общий алгоритм.

Теорема-АЛГОритм (Кан). Уравнение $U=\alpha n+\beta(n+1)+\gamma(n+p)$ разрешимо в чельх неотрицательных $\alpha, \beta, \gamma$ тогда и только тогда, когда

$$
\Theta(U-L(U)) \leqslant 0 .
$$

Здесь $q_{0}=n, q_{1}=n\{p / n\}, r_{0}=0, r_{1}=n+p$,

$$
\begin{gathered}
q_{j+1}=\left(\left[\frac{q_{j-1}-1}{q_{j}}\right]+1\right) q_{j}-q_{j-1}, \quad q_{j} \neq 0, \\
r_{j+1}=\left(\left[\frac{q_{j-1}-1}{q_{j}}\right]+1\right) r_{j}-r_{j-1}, \quad v=\min \left\{j \in \mathbb{N} \mid r_{j} \geqslant(n+1) q_{j}\right\}, \\
K(U)=q_{m-1}\left[\frac{n}{q_{m-1}}\left\{\frac{U}{n}\right\}\right], \quad q_{m}=0, \quad m \neq 1, \\
\varepsilon_{1}=\left[\frac{K(U)}{q_{1}}\right], \quad \varepsilon_{j+1}=\left[\frac{1}{q_{j+1}}\left(K(U)-\sum_{i=1}^{j} \varepsilon_{i} q_{i}\right)\right], \quad j=1,2,3, \ldots, m-2, \\
L(U)=\sum_{i=1}^{v-1} \varepsilon_{i} r_{i}, \quad L(U)=0, \quad m=1, \quad \Theta(U)=n\left\{\frac{U}{n}\right\}-\left[\frac{U}{n}\right] .
\end{gathered}
$$


СлЕДСтвиЕ 5. При выполнении (4) одним из решений будет следующее:

$$
\alpha=-\Theta(U-L(U)), \quad \beta=n\left\{\frac{U-L(U)}{n}\right\}, \quad \gamma=\frac{L(U)}{n+p} .
$$

Результаты работы докладьвались на семинаре “Дискретная математика" в Математическом институте им. В. А. Стеклова РАН 7 апреля 1997 года, а также на семинаре по теории чисел профессора А. Шинщеля в Институте математики Польской АН в августе 1997 года.

\section{СПИСОК ЦИТИРОВАННОЙ ЛИТЕРАТУРЫ}

1. Sylvester J. J. Mathematical questions, with their solutions // Educational Times. 1884. V. 41. P. 21. 2. Johnson S. M. // Canad. J. Math. 1960. V. 12. P. 390-398. 3. Stechkin B. S., Baranov V. I. Extremal Combinatorial Problems and Their Applications. Dordrecht: Kluwer Acad. Press, 1995. 4. Карацуба А.А., Офман Ю.П. // Докл. АН СССР. 1962. Т. 145. № 2. С. 293-294. 5. Шевченко В. Н. Качественные вопросы целочисленного программирования. М.: Физматлит, 1995.

Математический институт им. В. А. Стеклова РАН

Поступило

E-mail : boris@mi.ras.ru

20.05.97

Исправленный вариант 15.08 .97 